\title{
O futuro da nutrição parenteral no paciente crítico
}

The future of parenteral nutrition in critically ill patients

DOI: $10.37111 /$ braspenj.2020352013

Ivens Augusto Oliveira de Souza?

\section{Unitermos:}

Cuidados críticos. Nutrição parenteral. Resultados de cuidados críticos. Custos de cuidados de saúde.

\section{Keywords:}

Critical care. Parenteral nutrition. Critical care outcomes. Health care costs.

\section{Endereço de correspondência:}

Ivens Augusto Oliveira de Souza

Rua Joaquim Antunes, 810 - apto. 152 - Pinheiros -

São Paulo, SP, Brasil - CEP: 05415-001

E-mail: ivens_s@hotmail.com

\section{Submissão}

21 de maio de 2020

\section{Aceito para publicação}

10 de junho de 2020

\section{RESUMO}

O paciente crítico frequentemente apresenta disfunção do trato gastrintestinal, e comumente não atinge as suas necessidades nutricionais pelo trato gastrintestinal. Estudos recentes têm demonstrado que a nutrição parenteral é uma alternativa segura para o aporte nutricional desta população. $O$ objetivo desta revisão narrativa é descrever os principais riscos e cuidados que devem ser tomados durante o uso da nutrição parenteral no paciente crítico. A nutrição parenteral quando bem implementada, após a tentativa do uso do trato gastrintestinal, respeitando a fase aguda da doença crítica, guiada pela calorimetria indireta, e com uso de soluções balanceadas de lipídios, pode, inclusive, se associar a melhores desfechos, conforme sugerido por alguns estudos. Atualmente, existe uma maior demanda por fórmulas prontas de nutrição parenteral para uso visando agregar valor em saúde para os pacientes e as instituições.

\section{ABSTRACT}

Gastrointestinal dysfunction and inadequate nutrient intake are frequently seen in critically ill patients. Recent studies have shown that parenteral nutrition is safe among these patients. This narrative review aims to describe the risks associated with overfeeding and how to safely indicate and implement parenteral nutrition in the intensive care unit. After the acute phase of critical illness, individualized parenteral nutrition, using indirect calorimetry and balanced lipid emulsions, may be associated with better outcomes. The demand for ready-to-use bags has been growing intended to achieve the best value of care for patients and healthcare institutions.

1. Médico intensivista, Especialista em Medicina Intensiva pela Associação Brasileira de Medicina Intensiva (AMIB); Especialista em Terapia Nutricional Parenteral e Enteral pela Sociedade Brasileira de Nutrição Parenteral e Enteral (BRASPEN/SBNPE); Mestre em Ciências da Saúde pelo Instituto de Pesquisa do Hospital Sírio-Libanês, São Paulo, SP, Brasil; Gerente Médico - Produtos Hospitalares, Baxter Hospitalar Ltda., São Paulo, SP, Brasil. 


\section{INTRODUÇÃO}

A desnutrição hospitalar é uma condição frequente e está relacionada a maior morbi-mortalidade nos pacientes hospitalizados ${ }^{1,2}$. Uma recente revisão sistemática demonstrou que a desnutrição hospitalar na América Latina está presente em $40 \%$ a $60 \%$ dos pacientes, no momento da admissão hospitalar, e a prevalência aumenta quanto maior o tempo de internação'. Em pacientes críticos, a prevalência de desnutrição moderada a grave pode chegar a mais de $70 \%^{3}$, e esta população frequentemente não recebe todas as suas necessidades nutricionais durante a primeira semana de internação na unidade de terapia intensiva (UTI) ${ }^{4}$. Pacientes que evoluem com desnutrição hospitalar tendem a ter um tempo de internação mais prolongado ${ }^{2,3,5-9}$, maior número de complicações ${ }^{9-11}$ e readmissões ${ }^{3,8}$, maior mortalidade hospitalar $3,7,9$ e maior custo $2,6,9,12-14$. Um estudo brasileiro demonstrou que a desnutrição hospitalar aumentou em $308,5 \%$ o custo total da internação?.

A terapia nutricional é, portanto, primordial no tratamento do paciente hospitalizado e visa à manutenção ou à recuperação do estado nutricional do mesmo. Pacientes incapazes de ingerir todas as suas necessidades nutricionais pela via oral devem receber nutrição enteral e, quando o trato gastrointestinal não estiver funcionante, deve-se considerar o uso da nutrição parenteral total ou suplementar ${ }^{15-17}$.

\section{SEGURANÇA}

A nutrição parenteral, entretanto, não é isenta de riscos e estudos da década de 90 apontavam para um maior número de complicações infecciosas associadas ao uso da mesma, quando comparada à nutrição entera $\left.\right|^{18}$. Atualmente, a nutrição parenteral tem se tornado uma terapia cada vez mais segura ${ }^{19}$. Isso se deve a alguns fatores, como a maior preocupação em se evitar hiperalimentação, as medidas para minimizar o risco de síndrome de realimentação, o avanço no manejo dos cateteres vasculares, o controle glicêmico mais rígido e o uso de soluções balanceadas de lipídios ${ }^{19}$.

Recentemente, dois grandes estudos multicêntricos (CALORIES ${ }^{20}$ e NUTRIREA-2 ${ }^{21}$ ), randomizados e controlados foram publicados e incluíram, juntos, um total de 4.798 pacientes críticos, comparando o uso da nutrição parenteral com a nutrição enteral na primeira semana de UTI. Nenhum dos dois estudos demonstrou qualquer desfecho desfavorável associado ao uso da nutrição parenteral ${ }^{20,21}$. Inclusive, quando bem implementada, ou seja, após a tentativa do uso do trato gastrintestinal, no $4^{\circ}$ dia de internação na UTI, e guiada pela calorimetria indireta, a nutrição parenteral esteve associada a uma melhora dos parâmetros de imunidade e a uma diminuição do número de complicações infecciosas, como demonstrado nos estudos SPN ${ }^{22}$ e SPN2 ${ }^{23}$.

\section{INÍCIO}

Segundo as mais recentes diretrizes da American Society for Parenteral and Enteral Nutrition (A.S.P.E.N..) ${ }^{15}$, European
Society for Clinical Nutrition and Metabolism (ESPEN) ${ }^{16}$ e Sociedade Brasileira de Nutrição Parenteral e Enteral (BRASPEN) ${ }^{17}$, todo paciente crítico de alto risco nutricional deve receber a nutrição parenteral o quanto antes, se houver estabilidade clínica e uma contraindicação para o uso do trato gastrintestinal. Nos pacientes que não estão tolerando a progressão da nutrição enteral, o uso da nutrição parenteral suplementar está indicado após $\circ 7^{\circ}$ dia de internação na UTI ${ }^{15-17}$. De acordo com a ESPEN, o uso mais precoce da nutrição parenteral suplementar, entre $\circ 4^{\circ}$ e $7^{\circ}$ dia, deve ser avaliado caso a caso, e considerado em pacientes previamente desnutridos, de preferência guiado pela calorimetria indireta $^{16}$.

Em 2020, estas mesmas sociedades publicaram as recomendações para o tratamento nutricional do paciente crítico grave com COVID-19 e esta conduta foi reafirmada, com uma única ressalva: de que o limiar para o uso da nutrição parenteral suplementar nesta população deve ser mais baixo ${ }^{24-26}$. Trata-se de uma população vulnerável, frequentemente idosa e que comumente se apresenta com inapetência e sintomas gastrintestinais que levam à baixa aceitação alimentar na semana anterior à internação na UTI. Além disso, estes pacientes comumente necessitam de ventilação mecânica prolongada, muitas vezes em posição prona, o que dificulta o atingimento de todas as suas necessidades nutricionais pela via enteral. Sendo assim, a nutrição parenteral suplementar deve ser considerada em todo paciente que, após $\circ 5^{\circ}$ dia de internação na UTI, não atinja $65 \%$ das suas necessidades pela via enteral ${ }^{24-26}$.

\section{PROGRESSÃO}

Quanto à progressão da terapia nutricional, recentes estudos têm demonstrado de que esta deve ser lenta e gradual, tanto em relação às calorias quanto às proteínas ${ }^{27-30}$. Os principais riscos associados a uma prescrição agressiva das necessidades nutricionais desde o primeiro dia de internação na UTI são:

- Hiperalimentação - definida como a prescrição de mais de $100 \%$ do gasto energético basal do paciente, e que pode se manifestar com hiperglicemia, hipercapnia e hipertrigliceridemia ${ }^{29}$. Comumente relacionada ao uso de fórmulas preditivas para o cálculo das necessidades nutricionais, em serviços que não dispõem da calorimetria indireta $^{31,32}$;

- Produção endógena de calorias e hiperalimentação relativa - durante a fase aguda da doença crítica (primeiros 3-4 dias de internação na UTI), existe a ativação de um intenso processo inflamatório sistêmico e a liberação de hormônios contrarregulatórios, resultando na mobilização de substratos energéticos das reservas corporais dos pacientes. Acontece, portanto, uma produção endógena de calorias, que não é mensurada, e que não é bloqueada pela administração de nutrientes exógenos. Consequentemente, isto pode contribuir para uma hiperalimentação nos pacientes ${ }^{32,33}$; 
- Síndrome de realimentação - condição potencialmente fatal, desencadeada pela oferta de nutrientes, e que se manifesta com a queda de eletrólitos plasmáticos, principalmente fósforo, potássio e magnésio ${ }^{34}$. Um estudo retrospectivo demonstrou que a queda de fósforo até 72 horas após o início da terapia nutricional pode acontecer em quase $40 \%$ dos pacientes críticos em ventilação mecânica, mesmo que não haja nenhum fator de risco para esta manifestação ${ }^{35}$. Um outro estudo prospectivo, randomizado e controlado, com pacientes que apresentaram a síndrome de realimentação, demonstrou melhor sobrevida nos pacientes que receberam nutrição hipocalórica por pelo menos 48 horas antes de continuarem a progressão da oferta nutricional ${ }^{36}$;

- Calorias não nutricionais - o soro glicosado, o citrato usado na hemodiálise contínua e o propofol são exemplos de substâncias que contribuem para a oferta de calorias e que, frequentemente, são negligenciadas na dose total das necessidades nutricionais. Usando como exemplo o propofol, onde cada $\mathrm{ml}$ possui aproximadamente 1,1 kcal e 0,1 g de lipídio, um paciente recebendo $20 \mathrm{ml} / \mathrm{h}$ de propofol, ao final de 24 horas terá recebido $528 \mathrm{kcal}$ e $48 \mathrm{~g}$ de lipídio à base de óleo de soja ${ }^{37}$;

- Inibição de autofagia - a autofagia é um processo celular de reciclagem de organelas e outras estruturas disfuncionantes, além de materiais tóxicos acumulados dentro da célula, sendo extremamente importante na fase aguda da doença crítica, como um mecanismo de sobrevivência celular. A administração de nutrientes, principalmente de proteína, de forma agressiva, nesta fase, pode inibir este processo e impactar no desfecho dos pacientes ${ }^{38,39}$;

- Aumento da liberação de glucagon - um estudo clínico e experimental demonstrou que a administração de aminoácidos, na primeira semana de sepse, pode potencializar a liberação de glucagon e estimular maior catabolismo de aminoácidos no fígado, diminuindo a disponibilidade plasmática de aminoácidos ${ }^{40}$.

Sendo assim, ao invés de prescrever 100\% das necessidades nutricionais desde o primeiro dia, esta progressão deve ser feita aos poucos, durante 3 a 4 dias, respeitando a fase aguda da doença crítica ${ }^{27,28}$. A Figura 1 demonstra uma sugestão de progressão da terapia nutricional em relação às ofertas calórica e proteica, com base nas últimas recomendações e publicações científicas ${ }^{27,28}$.

\section{COMPOSIÇÃO}

A nutrição parenteral, quando indicada, deve conter todos os três macronutrientes: glicose, aminoácidos e lipídios; além dos micronutrientes: eletrólitos, vitaminas e elementos traço ${ }^{15-17}$.

A solução de glicose geralmente utilizada pela via central é a $50 \%$, e as soluções de aminoácidos disponíveis no mercado pouco diferem em termos de aminograma, mantendo uma concentração de $10 \%$ ou $15 \%$.

Com relação ao lipídio, deve-se optar por uma emulsão lipídica que interfira o mínimo possível na resposta inflamatória e imunológica do paciente. O óleo de soja ou o óleo de peixe puros devem ser evitados, pois interferem diretamente na resposta inflamatória do paciente, causando um estado pró-inflamatório ou anti-inflamatório, respectivamente. Ambos têm propriedades de imunossupressão. Sendo assim, todas as sociedades concordam que se deve dar preferência para soluções balanceadas de lipídios que contenham apenas um mínimo de óleo de soja suficiente para repor ácidos graxos essenciais ${ }^{15-17}$.

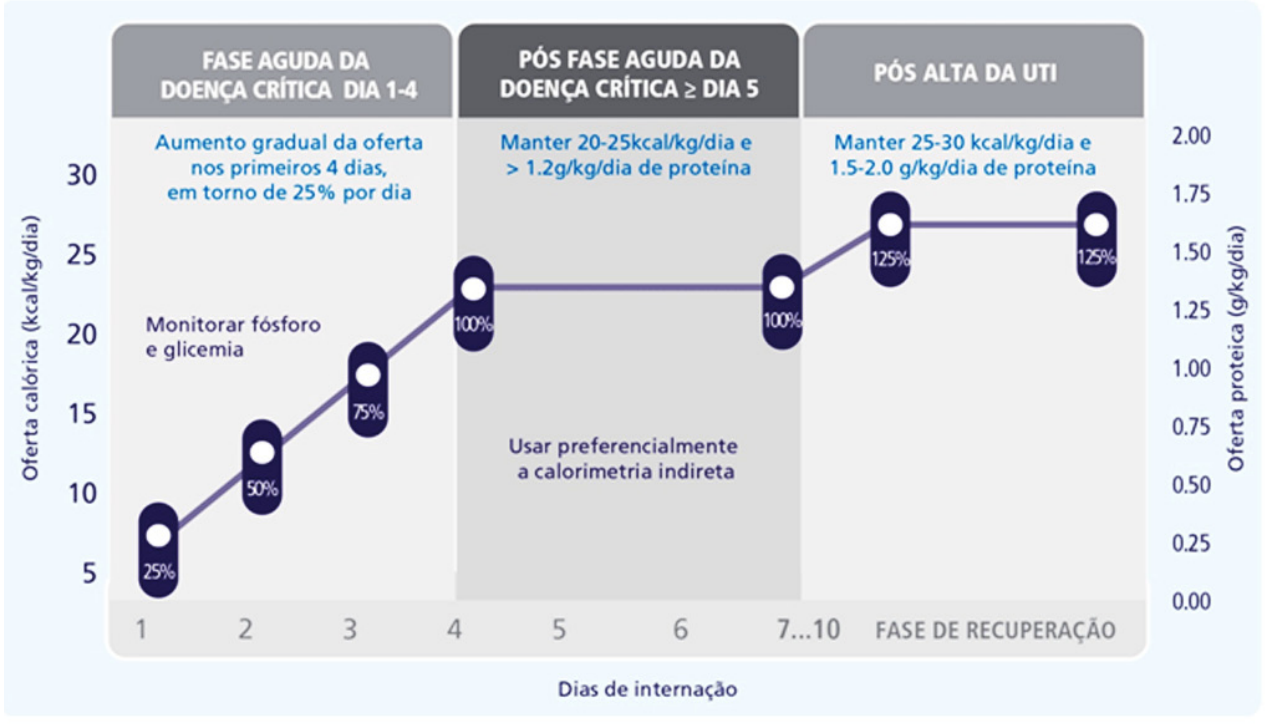

Figura 1 - Sugestão de progressão da terapia nutricional nos primeiros 10 dias de internação na UTI. Adaptado de Koekkoek \& van Zanten ${ }^{27}$ e van Zanten et al. ${ }^{28}$. 


\section{TIPO}

Tanto as nutrições parenterais prontas para uso (ou industrializadas) quanto as manipuladas podem ser utilizadas nos pacientes críticos ${ }^{15-17}$. A nutrição parenteral, quando individualizada, contém exatamente todas as necessidades nutricionais do paciente, além de permitir o acréscimo e a manipulação de todos os micronutrientes. Entretanto, há o risco de erros de prescrição, transcrição e manipulação, além do inconveniente de serem manipuladas fora da estrutura hospitalar, com uma demora considerável entre a prescrição pelo profissional e a administração ao paciente ${ }^{41}$. Além disso, a logística de transporte e o armazenamento de uma bolsa manipulada no hospital não são simples, pois exigem ambiente adequado e controle estrito de temperatura.

Recentemente, com o conceito de valor em medicina e os quatro pilares do "quadruple aim"42,43: melhor desfecho, menor custo, maior satisfação do paciente e melhor experiência do provedor de saúde, muitos hospitais têm migrado da utilização das nutrições parenterais manipuladas para as soluções prontas para uso. Os principais motivos que podem estar associados a esta escolha estão descritos no Quadro 1.

Quadro 1 - Principais motivos associados à preferência por bolsas prontas de nutrição parenteral.

\begin{tabular}{cl}
\hline Motivo & Explicação \\
\hline Praticidade na prescrição & $\begin{array}{l}\text { Por se tratar de uma bolsa pronta para uso, há menor necessidade de realização de cálculos, e elas } \\
\text { atingem as recomendações nutricionais para a maioria dos pacientes }\end{array}$
\end{tabular}

Redução de erros de prescrição, A quantidade de eletrólitos no interior de cada bolsa já é previamente estabelecida, sem risco de intranscrição e manipulação compatibilidades e precipitações

Redução no tempo e nos erros de checagem

Modelo atual de progressão da terapia nutricional

Reposição de eletrólitos

Reposição de vitaminas e oligoelementos

Redução no tempo de dispensação

Redução de desperdícios

Redução de custos

Garantia de qualidade e universalização do cuidado
As bolsas de nutrição parenteral individualizadas devem passar por, no mínimo, 4 processos de checagem de cada elemento prescrito, uma vez que não pode haver a possibilidade da administração da bolsa desenvolvida para um paciente em outro paciente. No caso de uma bolsa pronta para uso, basta a checagem do nome da bolsa, do volume a ser infundido e da velocidade de infusão prescrita

Uma vez que a progressão da terapia nutricional deve ser lenta e gradual, não existe uma necessidade formal de individualização na prescrição nos primeiros 3-4 dias de início da nutrição parenteral

Com a nutrição parenteral pronta para uso, toda reposição de eletrólitos deve ser feita em paralelo, o que garante maior agilidade quando comparada com a mesma reposição dentro de uma bolsa de nutrição parenteral, que será infundida em 24 horas. Cada vez mais existe uma preocupação com a síndrome de realimentação e a manutenção da homeostase plasmática em relação aos eletrólitos ${ }^{34}$

No caso das nutrições parenterais prontas para uso, essa reposição deve ser feita à parte, o que é uma vantagem, pois pode ser feita em baixo volume e em apenas 1 hora, minimizando o tempo de exposição e evitando a degradação pela luz ou pelo oxigênio ${ }^{44}$

A nutrição parenteral pronta para uso fica em estoque, dentro do hospital, armazenada à temperatura ambiente, com plena disponibilidade para o uso imediato após a prescrição. Dependendo da localização da farmácia de manipulação, as nutrições parenterais individualizadas podem demorar mais do que 8 horas para serem dispensadas aos pacientes, devido a todo o processo de checagem, manipulação, dispensação, transporte, recebimento e degelo

O paciente crítico é um paciente dinâmico e pode mudar sua condição clínica rapidamente durante o dia. Uma nutrição parenteral individualizada que foi prescrita pela manhã, por exemplo, pode não ser mais adequada para ser instalada no mesmo paciente no período da noite. Neste caso, a bolsa de nutrição parenteral deve ser descartada. 0 mesmo acontece quando há algum acidente com a bolsa de nutrição parenteral manipulada. Ademais, em ambas as situações, o paciente pode acabar ficando mais de 24 horas sem receber qualquer aporte nutricional

As realidades e os preços no Brasil variam bastante, porém existem situações em que o custo da nutrição parenteral manipulada é mais elevado do que a nutrição parenteral pronta para uso. Independente do preço do produto, devemos levar em consideração, também, a melhoria de processos, a carga de trabalho e o tempo dispendido pelos profissionais envolvidos ${ }^{41,45}$

As nutrições parenterais prontas para uso passam por um processo industrial de produção, são regulamentadas pela ANVISA e já saem de fábrica com laudo microbiológico. O Brasil, com uma área de $8.516 .000 \mathrm{~km}^{2}$, não dispõe de farmácias de manipulação com a estrutura complexa necessária em todos os municípios ou regiões. A nutrição parenteral pronta para uso faz com que cada paciente internado, onde quer que ele esteja, independente da condição social, possa receber uma terapia nutricional de qualidade, garantindo a universalização da terapia nutricional parenteral em todo o País e minimizando as mazelas da desnutrição hospitalar 
Embora o portfólio de nutrições parenterais prontas para uso atenda às necessidades da maioria dos pacientes, ainda não é tão amplo no Brasil como em outros países ou como o de fórmulas enterais. Por exemplo, pacientes com insuficiência renal evoluindo com distúrbios hidroeletrolíticos graves, pacientes necessitando de terapia dialíitica, pacientes obesos e pacientes com hipertrigliceridemia são situações em que deve haver uma individualização da terapia nutricional parenteral. As bolsas prontas possuem dose fixa de macronutrientes, além de conterem dose mínima diária de eletrólitos e, nesses casos, comumente é necessária a retirada de eletrólitos, um aporte proteico maior, a suspensão ou a redução dos lipídeos.

A indústria da nutrição parenteral, entretanto, está evoluindo graças à ciência e à inovação. Novas formulações estão sendo desenvolvidas com maior teor proteico, sem a adição de eletrólitos, em bolsas de diferentes volumes e que permitem a ativação de nutrientes específicos. Provavelmente, num futuro próximo, a nossa abordagem com a nutrição parenteral seja semelhante à da nutrição enteral.

\section{CONCLUSÃO}

O paciente crítico, frequentemente, apresenta disfunção do trato gastrintestinal e a nutrição parenteral configura como uma das principais alternativas para o suporte nutricional desta população. Recentes estudos têm demonstrado que se trata de uma prática segura, desde que bem indicada, adequadamente monitorada e guiada pela calorimetria indireta, idealmente. Tanto a nutrição parenteral manipulada quanto a industrializada são capazes de atingir as necessidades nutricionais dos pacientes e impactar em desfechos clínicos e na experiência do paciente. $\bigcirc$ uso de bolsas prontas para uso tem se tornado cada vez mais frequente, visando à melhoria de processos, à diminuição de custos e à maior satisfação do provedor de saúde.

\section{REFERÊNCIAS}

1. Correia MITD, Perman MI, Waitzberg DL. Hospital malnutrition in Latin America: a systematic review. Clin Nutr. 2017;36(4):958-67.

2. Norman K, Pichard C, Lochs H, Pirlich M. Prognostic impact of disease-related malnutrition. Clin Nutr. 2008;27(1):5-15.

3. Agarwal E, Ferguson M, Banks M, Batterham M, Bauer J, Capra $\mathrm{S}$, et al. Malnutrition and poor food intake are associated with prolonged hospital stay, frequent readmissions, and greater in-hospital mortality: results from the Nutrition Care Day Survey 2010. Clin Nutr. 2013;32(5):737-45.

4. Vallejo KP, Martínez CM, Adames AAM, Fuchs-Tarlovsky V, Nogales GCC, Paz RER, et al. Current clinical nutrition practices in critically ill patients in Latin America: a multinational observational study. Crit Care. 2017;21(1):227.

5. Heyland DK, Dhaliwal R, Wang M, Day AG. The prevalence of iatrogenic underfeeding in the nutritionally 'at-risk' critically ill patient: results of an international, multicenter, prospective study. Clin Nutr. 2015;34(4):659-66.

6. Chima CS, Barco K, Dewitt ML, Maeda M, Teran JC, Mullen KD. Relationship of nutritional status to length of stay, hospital costs, and discharge status of patients hospitalized in the medicine service. J Am Diet Assoc. 1997;97(9):975-80.

7. Goiburu ME, Goiburu MMJ, Bianco H, Díaz JR, Alderete F, Palacios MC, et al. The impact of malnutrition on morbidity, mortality and length of hospital stay in trauma patients. Nutr Hosp. 2006;21(5):604-10.

8. Lim SL, Ong KC, Chan YH, Loke WC, Ferguson M, Daniels L. Malnutrition and its impact on cost of hospitalization, length of stay, readmission and 3-year mortality. Clin Nutr. 2012;31(3):345-50.

9. Correia MITD, Waitzberg DL. The impact of malnutrition on morbidity, mortality, length of hospital stay and costs evaluated through a multivariate model analysis. Clin Nutr. 2003;22(3):235-9.

10. Haydock DA, Hill GL. Impaired wound healing in surgical patients with varying degrees of malnutrition. JPEN J Parenter Enteral Nutr. 1986;10(6):550-4.

11. Schneider SM, Veyres P, Pivot X, Soummer AM, Jambou P, Filippi J, et al. Malnutrition is an independent factor associated with nosocomial infections. Br J Nutr. 2004;92(1):105-11.

12. Reilly Jr JJ, Hull SF, Albert N, Waller A, Bringardener S. Economic impact of malnutrition: a model system for hospitalized patients. JPEN J Parenter Enteral Nutr. 1988;12(4):371-6.

13. Braunschweig C, Gomez S, Sheean PM. Impact of declines in nutritional status on outcomes in adult patients hospitalized for more than 7 days. J Am Diet Assoc. 2000;100(11):1316-24.

14. Curtis LJ, Bernier P, Jeejeebhoy K, Allard J, Duerksen D, Gramlichet L, al. Costs of hospital malnutrition. Clin Nutr. 2017;36(5):1391-6.

15. McClave SA, Taylor BE, Martindale RG, Warren MM, Johnson DR, Braunschweig C, et al. Guidelines for the Provision and Assessment of Nutrition Support Therapy in the Adult Critically Ill Patient: Society of Critical Care Medicine (SCCM) and American Society for Parenteral and Enteral Nutrition (A.S.P.E.N.). JPEN J Parenter Enteral Nutr. 2016;40(2):159-211.

16. Singer $P$, BlaserAR, Berger MM, Alhazzani W, Calder PC, Casaer MP, et al. ESPEN guideline on clinical nutrition in the intensive care unit. Clin Nutr. 2019;38(1):48-79.

17. Castro MG, Ribeiro PC, Souza IAO, Cunha HFR, Silva MHN, Rocha EEM, et al. Diretriz brasileira de terapia nutricional no paciente grave. BRASPEN J. 2018;33(Supl 1):2-36.

18. Elke G, van Zanten ARH, Lemieux M, McCall M, Jeejeebhoy KN, Kott M, et al. Enteral versus parenteral nutrition in critically ill patients: an updated systematic review and meta-analysis of randomized controlled trials. Crit Care. 2016;20(1):117.

19. Berger MM, Pichard C. Parenteral nutrition in the ICU: lessons learned over the past few years. Nutrition. 2019;59:188-94.

20. Harvey SE, Parrott F, Harrison DA, Bear DE, Segaran E, Beale $\mathrm{R}$, et al. CALORIES Trial Investigators. Trial of the route of early nutritional support in critically ill adults. N Engl J Med. 2014;371(18):1673-84.

21. Reignier J, Boisramé-Helms J, Brisard L, Lascarrou JB, Hssain AA, Anguel N, et al. NUTRIREA-2 Trial Investigators; Clinical Research in Intensive Care and Sepsis (CRICS) group. Enteral versus parenteral early nutrition in ventilated adults with shock: a randomised, controlled, multicentre, open-label, parallel-group study (NUTRIREA-2). Lancet. 2018;391(10116):133-43.

22. Heidegger CP, Berger MM, Graf S, Zingg W, Darmon P, Costanza $\mathrm{MC}$, et al. Optimisation of energy provision with supplemental parenteral nutrition in critically ill patients: a randomized controlled clinical trial. Lancet. 2013;381(9864):385-93.

23. Berger MM, Pantet O, Jacquelin-Ravel N, Charrière M, Schmidt S, Becce F, et al. Supplemental parenteral nutrition improves immunity with unchanged carbohydrate and protein metabolism 
in critically ill patients: the SPN2 randomized tracer study. Clin Nutr. 2019;38(5):2408-16.

24. Martindale R, Patel JJ, Taylor B, Warren M, McClave SA. Nutrition recommendations for caring for the critically ill patient with COVID-19. Joint Recommendations from SCCM and ASPEN. Nutrition therapy in the patient with COVID-19 disease requiring ICU care. Published online 2020 Apr 1. [cited 2020 May 20]. Available from: https:// www.sccm.org/COVID19RapidResources/Resources/ Nutrition-Therapy-in-the-Patient-with-COVID-19-Dis

25. Barazzoni R, Bischoff SC, Breda J, Wickramasinghe K, Krznaric Z, Nitzan D, et al. ESPEN expert statements and practical guidance for nutritional management of individuals with SARSCoV-2 infection. Clin Nutr. 2020;39(6):1631-8.

26. Campos LF, Barreto PA, Ceniccola GD, Gonçalves RC, Matos LBN, Zambelli CMSF, et al. Parecer BRASPEN/AMIB para o enfrentamento da COVID-19 em pacientes hospitalizados. BRASPEN J. 2020;35(1):3-5.

27. Koekkoek KWAC, van Zanten ARH. Nutrition in the ICU: new trends versus old-fashioned standard enteral feeding? Curr Opin Anaesthesiol. 2018;31(2):136-43.

28. van Zanten ARH, De Waele E, Wischmeyer PE. Nutrition therapy and critical illness: practical guidance for the ICU, post-ICU, and long-term convalescence phases. Crit Care. 2019;23(1):368.

29. Koekkoek WACK, van Setten CHC, Olthof LE, Kars JCNH, van Zanten ARH. Timing of PROTein INtake and clinical outcomes of adult critically ill patients on prolonged mechanical VENTilation: the PROTINVENT retrospective study. Clin Nutr. 2019;38(2):883-90.

30. Preiser JC. High protein intake during the early phase of critical illness: yes or no? Crit Care. 2018;22(1):261.

31. McClave SA, Lowen CC, Kleber MJ, Nicholson JF, Jimmerson SC, McConnell JW, et al. Are patients fed appropriately according to their caloric requirements? JPEN J Parenter Enteral Nutr. 1998;22(6):375-81.

32. Berger MM, Pichard C. Feeding should be individualized in the critically ill patients. Curr Opin Crit Care. 2019;25(4):307-13.

33. Oshima T, Heidegger CP, Pichard C. Supplemental parenteral nutrition is the key to prevent energy deficits in critically ill patients. Nutr Clin Pract. 2016;31(4):432-7.

34. Boot R, Koekkoek KWAC, van Zanten ARH. Refeeding syndrome: relevance for the critically ill patient. Curr Opin Crit Care. 2018;24(4):235-40.
35. Olthof LE, Koekkoek WACK, van Setten C, Kars JCN, van Blokland D, van Zanten ARH. Impact of caloric intake in critically ill patients with, and without, refeeding syndrome: a retrospective study. Clin Nutr. 2018;37(5):1609-17.

36. Doig GS, Simpson F, Heighes PT, Bellomo R, Chesher D, Caterson ID, et al. Restricted versus continued standard caloric intake during the management of refeeding syndrome in critically ill adults: a randomised, parallel-group, multicentre, single-blind controlled trial. Lancet Respir Med. 2015;3(12):943-52.

37. Bousie E, van Blokland D, Lammers HJ, van Zanten AR. Relevance of non-nutritional calories in mechanically ventilated critically ill patients. Eur J Clin Nutr. 2016;70(12):1443-50.

38. Hermans G, Casaer MP, Clerckx B, Güiza F, Vanhullebusch $\mathrm{T}$, Derde $\mathrm{S}$, et al. Effect of tolerating macronutrient deficit on the development of intensive-care unit acquired weakness: a subanalysis of the EPaNIC trial. Lancet Respir Med. 2013;1(8):621-9.

39. Casaer MP, Wilmer A, Hermans G, Wouters PJ, Mesotten D, Van den Berghe G. Role of disease and macronutrient dose in the randomized controlled EPaNIC trial: a post hoc analysis. Am J Respir Crit Care Med. 2013;187(3):247-55.

40. Thiessen SE, Derde S, Derese I, Dufour T, Vega CA, Langouche $\mathrm{L}$, et al. Role of glucagon in catabolism and muscle wasting of critical illness and modulation by nutrition. Am J Respir Crit Care Med. 2017;196(9):1131-43.

41. Genton L, Mühlebach S, Dupertuis YM, Pichard C. Ergonomic and economic aspects of total parenteral nutrition. Curr Opin Clin Nutr Metab Care. 2006;9(2):149-54.

42. Bodenheimer T, Sinsky C. From triple to quadruple aim: care of the patient requires care of the provider. Ann Fam Med. 2014;12(6):573-6.

43. Barnett KAG. In pursuit of the fourth aim in health care: the joy of practice. Med Clin North Am. 2017;101(5):1031-40.

44. Blaauw R, Osland E, Sriram K, Ali A, Allard JP, Ball P, et al. Parenteral provision of micronutrients to adult patients: an expert consensus paper. JPEN J Parenter Enteral Nutr. 2019;43(Suppl 1):S5-S23.

45.Yu J, Wu G, Tang Y, Ye Y, Zhang Z. Efficacy, safety, and preparation of standardized parenteral nutrition regimens: threechamber bags vs compounded monobags - a prospective, multicenter, randomized, single-blind clinical trial. Nutr Clin Pract. 2017;32(4):545-51.

Local de realização do estudo: Hospital Sírio-Libanês, São Paulo, SP, Brasil.

Conflito de interesse: $\mathrm{O}$ autor já recebeu honorários para palestra e/ou consultoria das seguintes empresas: Danone Nutrícia, Nestle Health Science, Fresenius Kabi, Farmoterápica, Abbott, Prodiet Medical Nutrition e Baxter Hospitalar. Atualmente, atua como Gerente Médico de Produtos Hospitalares na Baxter Hospitalar, São Paulo, SP, Brasil. 